\title{
REJOUER LES SAVOIRS ANTHROPOLOGIQUES: DE DURKHEIM AUX ABORIGÈNES ${ }^{*}$
}

\author{
Barbara Glowczewski \\ Laboratoire d'Anthropologie sociale (CNRS/EHESS/Collège de France) - \\ France
}

Résumé: Les formes élémentaires de la vie religieuse de Durkheim (2013) s'appuient essentiellement sur les observations et analyses de Spencer et Gillen (1899) des rituels d'Australie centrale. Découlant de 35 ans de recherches en Australie, l'article montre que le paradigme du XXe siècle a empêché Durkheim de voir l'importance du rapport à la terre dans la cosmologie et les pratiques rituelles des Aborigènes. Il a aussi ignoré le dynamisme réticulaire de leurs cartographies totémiques que, depuis la colonisation, ils continuent à réactualiser par l'art et les luttes sociales. La réappropriation indigène par la parole et d'autres expressions de leurs propres systèmes de savoir pose la question de la légitimité contemporaine des interprétations anciennes. Patrimonialisés, Durkheim et d'autres deviennent des mythes fondateurs des sciences sociales qui s'opposent parfois à la reconnaissance des peuples concernés. L'anthropologie est ainsi confrontée à un problème à la fois éthique et politique.

Mots-clés: Australie, cartographies totémiques, patrimonialisation, réappropriations autochtones.

Abstract: The elementary forms of religious life by Durkheim (2013) largely draw on Spencer and Gillen's observations and analysis of Central Australian rituals. Stemming from 35 years of research across Australia, this article shows that the paradigm of the XXe century has prevented Durkheim to see the importance of the relation to land in the cosmology and ritual practices of Aboriginal people. This paradigm also ignored the reticular dynamism of their totemic cartographies that since colonization

* Une version courte de ce texte a été présentée au Colloque « Les formes élémentaires de la vie religieuse de Durkheim. Perspectives pour l'anthropologie », (6-8 juin 2012) célébrant le centenaire du livre, à la session d'ouverture « Ethnographie et théorie », École Normale Supérieure (Ulm), organisé par Perig Pitrou et Frédéric Keck, en collaboration avec le musée du quai Branly et le Collège de France.

Horizontes Antropológicos, Porto Alegre, ano 20, n. 41, p. 381-403, jan./jun. 2014 http://dx.doi.org/10.1590/S0104-71832014000100014 
Indigenous Australians have been reactualizing through art and social struggles. The Indigenous empowerment through speech and other expressions of their systems of knowledge questions the contemporary legitimacy of ancient interpretations. While Durkheim and others are "patrimonialized" into Western heritage, they become foundation myths of social sciences which are sometimes opposed to the recognition of the people they studied. Consequently anthropology is confronted to an ethical and political problem.

Keywords: Australia, indigenous empowerment, patrimonialization, totemic cartographies.

The Elementary Forms was created in an effort to answer Spencer and Gillen, and to glue society and religion together again. In the process, it often misrepresented their account, yet without amounting to a total

falsification of their ethnography. It is instead an imaginative reconstruction, which involved its author in developing a whole new seminal theory of his own. The work is both a transfiguration of Spencer and Gillen's Australia and a transfiguration of the old Durkheimian Australia.

(Watts Miller, 2012)

Durkheim est-il bon à penser pour les Aborigènes d'aujourd'hui? La question est à la fois théorique, pragmatique et politique. En effet, si les Aborigènes furent « bon à penser » les sciences sociales depuis leur création, celles-ci furent secouées ces trente dernières années par l'introduction de l'histoire dans la théorie anthropologique, particulièrement celle des populations considérées jusque là « sans histoire », alors que leur histoire est non seulement orale mais aussi constituée d'une multitude d'archives visuelles et matérielles précoloniales. La question interroge ainsi d'une part le statut de Durkheim comme un des " mythes » fondateurs des sciences sociales et d'autre part le statut de la prise de parole des populations étudiées au regard de l'histoire de l'anthropologie et des observations ethnographiques contemporaines.

Les données sur les peuples premiers d'Australie ont contribué aux fondements des sciences sociales, depuis Durkheim et Mauss à Lévi-Strauss, en passant par Freud (1975). Or la prise de parole et les activités aborigènes des dernières décennies- telles les innovations rituelles, les luttes pour la reconnaissance de leurs sites sacrés et les peintures totémiques sur toile - remettent

Horizontes Antropológicos, Porto Alegre, ano 20, n. 41, p. 381-403, jan./jun. 2014 
en question certains des paradigmes fondés sur les anciennes interprétations du totémisme. Face à la colonisation, les Aborigènes se sont battus pour acquérir des droits à la citoyenneté australienne, mais, qu'ils vivent dans des communautés reculées du désert, des rivières et des côtes du nord, ou bien en ville, la majorité continue de résister aux nouvelles formes d'assimilation forcée, ou de rejet stigmatisant, en insistant sur leurs particularités culturelles et ontologiques, même si beaucoup sont métissés depuis des générations. L'histoire particulière de l'Australie qui, sous prétexte de métissage et de politique de " blanchiment », a séparé entre 1905 et les années 1970 un enfant sur cinq de leurs parents, pour les éloigner de leur milieu aborigène, a créé ce paradoxe : la souffrance du déni colonial des origines a suscité un mouvement de revendication de telles origines et un refus de reconnaissance du métissage. Nommer les degrés de métissage est considéré comme une démarche coloniale dénigrante de l'intégrité des personnes qui choisissent de s'identifier comme Aborigènes et Black, " noires ", quelle que soit leur couleur de peau. L’Aboriginalité concerne tous les descendants : en ce sens elle n'est pas essentialiste mais construite par diverses expériences d'héritage tant culturel qu'historique, qui impliquent souvent un partage de souffrance, de rejet, de résistance et de créativité.

Nous allons voir que les " éléments " aborigènes qui insistent sur leur spécificité et persistent dans leurs modes d'existence actuels comme traits de leur singularité ne semblent pas correspondre aux «formes élémentaires » dégagées par Durkheim. A ce titre, je ne pense pas que ses interprétations des Aborigènes puissent nous aider à comprendre leur singularité ou les questions que les religions posent aujourd'hui; je doute même qu'elles éclairent le système spirituel et la société aborigène de la fin du XIXe siècle. En revanche, j’ai pour hypothèse que certains agencements qui caractérisent la spiritualité aborigène contemporaine - $\mathrm{y}$ compris la manière dont ils rejettent ou absorbent la christianisme - nous aident à comprendre quelque chose de leur perception que j'appelle « réticulaire » et qui, malgré les aléas de la colonisation, continue à mettre en lien toutes les dimensions de la vie. Des formes transversales à l'humanité se dégagent de bien des études des peuples autochtones d'Australie mais aussi d'ailleurs, qui déplacent les questions de Durkheim, en sortant l'humanité de ses catégories exclusives - culture/nature, individu/société, corps/esprit, imaginaire/réel - pour appréhender l'humain dans un projet écosophique, où se nouent, au sens de Félix Guattari, ce qu'il appelait trois 
écologies : environnementale (à la fois nature et technique), sociale et mentale, un nouveau paradigme à la fois esthétique, éthique et politique (Guattari, 1989; Guattari; Rolnik, 1986).

\section{Totémisme ou Dreamings : des classifications aux réseaux}

La réinterprétation des cultures traditionnelles par elles-mêmes n'est pas nouvelle : pour beaucoup de peuples vivant de chasse et de cueillette, elle a été le propre même de la survie, consistant, par exemple, à toujours redonner du sens aux pratiques quotidiennes et aux événements ponctuant les vies individuelles et collectives. La nouveauté vient de la confrontation souvent violente avec l’Occident qui, ayant voulu les assimiler en détruisant leur spécificité, produit maintenant un discours qui cherche à assimiler leurs productions artistiques et musicales dans l'histoire de l'art universel tout en leur déniant l'authenticité de leur être social.

Les voix des peuples autochtones sont éditées ici et là [...] Mais le discours des autochtones sur eux-mêmes n’a pas droit de cité partout. (Glowczewski, 2004, p. 22).

Les Aborigènes se sont adaptés aux moyens de leur époque - les radios, les journaux, l'art, la vidéo, le cinéma, l'internet, et la politique - afin de mettre en scène leurs valeurs et aussi diffuser leurs critiques épistémologiques de la manière dont ils sont stéréotypés et souvent discriminés. Ces stéréotypes, qui s'appuient sur des archétypes évolutionnistes au fondement de nos disciplines, ne relèvent pas que du passé colonial : ils guident encore les gouvernements, les médias et certains de nos collègues. Malgré le scepticisme des nostalgiques de l'authenticité supposée perdue des sauvages, bien des communautés aborigènes- en créant des coopératives d'art dans les années 1970 et 1980 - ont réussi à faire reconnaître la singularité culturelle de mouvements artistiques locaux qui, depuis trente ans, renouvellent régulièrement le marché mondial de l'art contemporain avec des œuvres qui ne finissent pas d'étonner. Initié par des artistes de Papunya (Myers, 2002), l'art des peintures dites « à points » (dot paintings) réalisées sur des toiles avec des couleurs à l'acrylique, démontre la manière cartographique dont les Aborigènes du désert vivent encore ce que les anthropologues ont appelé le totémisme et que le monde de l'art appelle " Dreamings ", Rêves, ou plutôt comme l'indique la forme progressive en anglais : « ce qui est en train de rêver ». 
Le terme « Dreaming » est une traduction, qui fut proposée par Spencer et Gillen, de concepts cosmologiques des Arrernte (Aranda, Arunta) et de leur voisins qui désignent par ce terme à la fois des êtres totémiques, les récits mythiques qui racontent leurs voyages et les itinéraires géographiques qui relient les sites sacrés qu'ils ont marqués sur leur passage. Dreaming désigne aussi le temps de référence de ces actions ancestrales que j’ai proposé de traduire par " espace-temps du Rêve » plutôt que « temps du Rêve ", afin d'insister sur le lien des totems non seulement avec un temps supposé mythique mais aussi un espace à la fois physique et virtuel, en devenir dans toutes ses manifestations d'existence, y compris les hommes (Glowczewski, 1991). L'usage de Dreaming a rencontré un succès mondial qui a créé autant de malentendus que le terme « totem » emprunté aux Algonkins d'Amérique. Mais il reste que l'aspect totémique des Dreamings aborigènes le plus revendiqué aujourd'hui - celui de liens spirituels avec des sites sacrés -, bien qu’évoqué par Spencer et Gillen, fut à peine commenté par Durkheim. Il remarqua dans une note que les totems pouvaient être des lieux mais sans mesurer l'importance de ces processus d'identification entre des lieux différents et des personnes, hommes ou femmes, qui sont reconnus come les manifestations en devenir de plusieurs sites totémiques reliés par des itinéraires, et à ce titre dotés à la fois de responsabilités rituelles et de droits fonciers qui les lient à d'autre dans des réseaux complexes et non pas des clans fermés.

J'ai pour hypothèse que le contexte de pensée de l'époque ne lui permettait pas - comme à beaucoup d'autres - de percevoir l'importance sociale, politique et existentielle de ces liens fonciers ni même de visualiser le modèle réticulaire dans lequel ils s'expriment. En effet, c'est l'enjeu politique de la lutte pour leur autodétermination qui a permis aux Aborigènes de surligner l'importance de leurs liens à la terre en tant qu'autochtones et donc propriétaires légitimes de terres dont ils avaient été spoliés par la colonisation. Mais c'est aussi un changement de paradigme occidental qui nous a permis de voir et symboliser l'ethnographie australienne autrement, dès lors que la réticularité de la projection des totems dans l'espace est devenue intuitivement plus perceptible, notamment parce que depuis l'internet, nous avons une pratique quotidienne des réseaux (Glowczewski, 2004, 2005, 2007).

La lecture faite par Durkheim, et d'autres après lui, des données aborigènes disponibles, réduisit les descriptions minutieuses de Spencer et Gillen, ainsi que de quelques autres observateurs de terrain, à des questions 
de classification et de religion, qui relevaient du paradigme classificatoire et dualiste de leur époque, opposant individu et société, nature et culture, corps et âme, etc. En témoigne notamment les débats sur les " esprits-enfants » aborigènes (totems dits de conception) dont l'interprétation était biaisée par la théologie de l'immaculée conception : le dualisme du corps et de l'esprit semblaient empêcher des théoriciens de l'époque (et même encore certains aujourd'hui) de concevoir que les Aborigènes puissent reconnaître le rôle de l'acte sexuel dans la reproduction tout en affirmant que la conception d'un enfant nécessite qu'un esprit-enfant se choisisse une mère et un père, et annonce sa conception dans un rêve que font soit les parents, soit des membres de la parentèle. La psychanalyse nous a habitués à relativiser ce dualisme du corps et de l'esprit : de multiples facteurs peuvent entrer en jeu pour faire un enfant, le rapport sexuel ne suffit pas. Un des enjeux des rêves d'annonciation de conception qui s'énoncent en termes de désirs et parfois de conflit comme une pulsion de rêve- est de reconnaître une autonomie à l'enfant, exprimée par son totem dit de conception, à la fois un nom totémique, un prénom (vers de chant condensé) et un lieu. Ce totem de conception (ou Dreaming de conception, les Walrpiri disent kurruwalpa) entre dans une constellation d'autres identifications totémiques (images forces kuruwarri) qui constituent le territoire existentiel particulier d'une personne dont les agencements cartographiques se recomposent au cours de sa vie et de ses interactions avec d'autres : processus exemplaire de la microsociologie de Tarde, souligné par Deleuze (1986) :

Tarde disait ceci : un courant d'imitation ou de propagation ne va pas d'un individu à un autre individu. Il va d'où à où? Il va d'un état de croyance à un état de croyance ou d'un état de désir à un état de désir. [...] Ça devient une très grande idée qui n’a rien à voir avec de la psychologie, mais qui a bien à voir avec de la microsociologie. Les croyances et les désirs sont les corpuscules sociaux. Vous voyez la force de la critique contre Durkheim : Durkheim en reste aux représentations, il ne voit pas ce qu'il y a sous la représentation. Ce qu'il y a sous la représentation... La représentation est un grand ensemble, c'est une instance molaire. Sous les représentations, il y a les corpuscules de croyance et de désir et les corpuscules de croyance et de désir sont inséparables d'ondes de propagation et l'onde de propagation de la croyance et du désir, c'est l'imitation [...] l'imitation et l'invention chez Tarde correspondent tout à fait - vous ne pouvez comprendre ce que je veux dire que un peu plus tard - correspondent tout à fait à ce que Foucault appelle des rapports de force.

Horizontes Antropológicos, Porto Alegre, ano 20, n. 41, p. 381-403, jan./jun. 2014 
Deleuze (1986) ajoute qu'il y eu un débat similaire entre Claude LéviStrauss et Edmund Leach, le premier fabriquant une macrosociologie des échanges avec des structures molaires et le second, lui opposant des pratiques effectives, un réseau latéral, transversal, en perpétuel instabilité. Le débat qui opposa en 1903 Durkheim à Tarde témoigne de l'arbitraire classificatoire et du primat du symbolique (représentations collectives) qui s’est imposé en France dans une certaine filiation dominante des théories en sciences sociales. En écoutant sur le net leur débat rejoué mot pour mot par les philosophes Bruno Karsenti et Bruno Latour dans un séminaire aux USA, ${ }^{1}$ on peut s'étonner que les étudiants du début du XX siècle devaient étudier et discuter leurs arguments opposés à cette époque, alors que l'histoire de nos disciplines a ensuite, pendant des décennies, préféré enterrer Tarde. Or les travaux de Gilbert Simondon (1964), Deleuze et Guattari (1980), puis Bruno Latour (2002) ou Maurizio Lazaratto $(1999,2002)$ ont montré à quel point la vision en flux de Tarde permet de mieux appréhender les phénomènes sociaux et économiques de notre temps, en sortant notamment de l'opposition entre individu et société. Selon Tarde, lors de ce débat qui l’opposa à Durkheim en 1903, des variétés individuelles, des innovations et des lois de l'invention se dégage « une résultante collective presque constante qui donne lieu à l'illusion ontologique de Monsieur Durkheim » du « fait social » qui substitue « milieu fantôme » aux relations toujours fluctuantes.

Bien que Durkheim ait gagné contre Tarde dans l'histoire patrimonialisée de la sociologie, Tarde revient en force, comme le montre les travaux de Latour (2012, p. 13), critiquant Durkheim :

Chose surprenante dans un livre qui annonce le passage des formes élémentaires évoluant, du moins le suppose-t-on, vers des formes plus évoluées, aucune transformation historique ne vient marquer la position faite à l'individu : les Aborigènes sont supposés bénéficier exactement du même appareillage psychologique que le révolutionnaire en bonnet phrygien de 1789 ou le sujet contemporain. Cette absence d'historicité prouve à quel point l'ouvrage est animé par un problème que les données ethnographiques ne sauraient aucunement éclairer.

1 http://www.bruno-latour.fr/fr/node/435 : d'après des recherches originales d'Eduardo Vlana Varga et de Louise Salmon, mise en scène de F. Ait-Touati, film de Martin Pavloff; http://anthem-group.net/tag/ bruno-karsenti/.

Horizontes Antropológicos, Porto Alegre, ano 20, n. 41, p. 381-403, jan./jun. 2014 
Les données ethnographiques anciennes - tordues par les débats du XIXe siècle et du XXe- résonnent autrement avec l'ethnographie plus récente et le monde d'aujourd'hui qui, notamment depuis l'avènement d'internet, se pense en flux et réseaux dynamiques. Mais il reste que si Tarde mettait en avant l'invention morale et la sociabilité, sans partager la vision quasi messianique d'une supposée amélioration du monde par la science - comme recherche de preuves pour éprouver des faits selon Durkheim -, il était un homme de son époque modelé par la tendance quasi générale des humanistes à n’avoir guère de recul sur la colonisation qui invoquait la nécessité de dépasser la supposée « arriération » des peuples colonisés au nom du progrès. Dès 1885, Clemenceau s"était lui élevé contre Le discours sur les «droits et devoirs des races supérieures » à « civiliser les races inférieures » de Jules Ferry. ${ }^{2}$

Le dernier siècle, et particulièrement le tournant de ce millénaire, a démontré l'échec partiel de la course en avant du progrès, les limites supposées civilisatrices de l'Occident, et la redécouverte de certains savoirs et pratiques des cultures anciennes comme valeurs contemporaines, non seulement à préserver au nom du patrimoine mondial, mais aussi à exploiter pour résoudre des problèmes contemporains. En témoigne, par exemple, les savoirs sur les plantes médicinales et certaines techniques chamaniques popularisées par les thérapeutes contemporains. D’une manière générale, cette reconnaissance de perspectives non occidentales nous invite à décoloniser la pensée et nos disciplines (Glowczewski, 2012). ${ }^{3}$

\footnotetext{
« Je répète qu'il y a pour les races supérieures un droit, parce qu’il y a un devoir pour elles. Elles ont le devoir de civiliser les races inférieures. » dit Jules Ferry en 1885 : « Je ne comprends pas que nous n'ayons pas été unanimes ici à nous lever d'un seul bond pour protester violemment contre vos paroles. Non, il n’y a pas de droit des nations dites supérieures contre les nations inférieures », lui répondit alors le député Clémenceau.

3 Voir à ce propos un échange filmé avec Eduardo Viveiros de Castro : Glowczewski B. (12 juillet 2011) : « Décoloniser l'anthropologie : agencements et réseaux existentiels des peuples autochtones. », Décolonisations de la pensée. Anthropologie, philosophie et politique. (2) leçons deleuzo-guatariennes », Journées Erraphis-Europhilosophie, Université de Toulouse : http://choplair.com.free.fr/ Europhilosophie/FIPS_videos/player.php?id=2011_12juil_glowczewski\&auto=1. Voir aussi, deux autres conférences filmées de Glowczewski B. (26 avril 2013) : « Décoloniser l’anthropologie : exemples australiens et français » (traduction en portugais par Claudia Fonseca), Porto Alegre, PPGAS/UFRGS Department seminar : https://vimeo.com/65924766; (12 août 2013), « Ethics of anthropological archives : academic heritage and Indigenous priorities », Canberra, AIATSIS : http://vimeo.com/73112943.
}

Horizontes Antropológicos, Porto Alegre, ano 20, n. 41, p. 381-403, jan./jun. 2014 


\section{Terrains et cartographies aborigènes}

Je suis partie sur le terrain australien en 1979 nourrie d'une approche poststructuraliste telle qu'enseignée au département d'ethnologie de Robert Jaulin, avec Michel De Certeau ou Jean-Toussaint Desanti à Paris 7-Jussieu, et par Deleuze, les cinéastes expérimentaux ou encore les féministes à Paris 8-Vincennes. La participation quasi quotidienne pendant cinq mois de danses, chants et peintures corporelles - mis en œuvre dans des rituels souvent séparés pour les femmes et les hommes mais qui célébraient les mêmes héros totémiques-, me permit de découvrir une manière d'être collective en constante performativité. Le totémisme n'était pas une simple affaire de classification mais un processus en devenir dans son actualisation rituelle sans cesse rejouée à travers un jeu de rôles où chacun était maître (boss) de certains totems/Rêves et assistant (worker/manager) d'autres : maître (kirda) de totems/Rêves qu'il ou elle appelait à la fois " père » et " frère/soeur » et assistant (kurdungurlu) de totems/rêves appelées soit mère soit « conjoint».

Ces rôles rituels étaient déterminés par la parenté dite de " classes » ou à 8 sous-sections appelées "noms de peau » (skin names) par les Warlpiri et leurs voisins du désert, tels les Aranda (Arrernte). De multiples équivalences et torsions étaient sans cesse en jeu afin de permettre aux gens de se situer les uns par rapport aux autres même s'ils n'étaient pas de la même famille ou alliés : toutes les relations se traduisaient en règles idéales de parenté que, bien sur, on transgressait souvent mais dont la logique systémique (porté par un cube ou groupe diédrique) assurait une certaine codification des rôles au regard des différentes choses nommées dans la nature et la culture qui couvraient l'ensemble du réseau totémique et du territoire tribal. Ce système était dynamique et semblait se substituer à l'émergence de chefs. Les "boss » rituels changeaient selon les totems célébrés. Les maîtres d'un totem donné ne pouvaient rien faire sans des assistants rituels qui détenaient la loi et certains savoirs. Cette relation s’inversait selon les totems et terres associés célébrées. Les maîtres d'une cérémonie devenaient les assistants des maîtres d'autres totems et terres lorsque ceux-ci célébraient leur rituel.

Il en allait de même entre les hommes et les femmes : les secrets et rituels des uns étaient complémentaires des secrets et rituels des autres. L'exclusion rituelle de chaque genre travaillait à produire une androgynie symbolique :

Horizontes Antropológicos, Porto Alegre, ano 20, n. 41, p. 381-403, jan./jun. 2014 
ce que j’ai appelé des « hyperfemmes » et des « hyperhommes », le modèle d'identification des femmes aux diverses femmes-hommes mythiques n'étant pas la même chose que celui des hommes aux hommes-femmes mythiques (Glowczewski, 1991). Les modèles mythiques étant ici ces êtres totémiques de « rêve ", pistes en devenir rejouées à chaque rituel.

Le territoire tribal - dont hommes et femmes avaient le gardiennage rituel - se déployait comme un réseau de lignes qui s’entrecroisaient en distribuant à tous des droits et des obligations rituelles spécifiques sur des séries de lieux reliés en fonction des identifications multiples de chacun -collectives et individuelles, tel l'esprit totémique de conception ou les totems hérités du groupe paternel, par adoption ou d'autres occasions. Un Warlpiri disait par exemple qu'il ou elle EST à la fois Opossum, Prune et Graine d'acacia, et aussi qu'il EST tel ou tel lieu, source ou rocher, où les êtres Opossum, Prune ou Graine ont laissé soit leur empreinte soit une partie ou une excrétion métamorphosée de leur corps. Chaque naissance appelait de nouvelles interprétations des liens anciens alors que les tabous funéraires obligeaient à ne plus énoncer certaines associations totémiques entre les lieux. Si certains rituels devenaient tabous le temps du deuil, de nouveaux émergeaient avec la révélation onirique de chants et peintures corporelles pensés comme des remémorations. Ces rêves réactualisaient dans les rituels les liens entre les Dreamings/totems et les sites, créant ainsi une cartographie dynamique : les séquences de récits chantés qui relient en ligne des centaines de lieux totémiques nommés se sont ainsi renouvelées au cours du temps au rythme des morts, des naissances et de l'interprétation des rêves.

Lorsque Félix Guattari lut ma thèse de $3^{\text {e }}$ cycle soutenue en 1982 après deux séjours de 5 mois en Australie, il remarqua que mes données et l'analyse que j'en proposais lui évoquaient Tarde plutôt que Durkheim, et il trouva là un exemple d'agencements collectifs et de production de territoires existentiels, et d'affects a-signifiants s'articulant dans les cartographies schizoanalytiques qu’il élaborait à l'époque (Glowczewski, 2011b ; Guattari, 1992 ; Guattari ; Glowczewski, 1987). J'étais partie en Australie, après une maîtrise sur les cinq sens et des films expérimentaux à la recherche d'une perception sensorielle qui ne passe pas par la représentation. J'avais lu que les veuves aborigènes (endeuillées dès le plus jeune âge en raison du mariage des petites filles à des hommes jusque trente ans plus âgées qu'elles) étaient soumises à un 
tabou de silence pendant au moins deux ans en vivant ensemble dans un camp qui leur était réservé, et j'avais postulé qu’elles devaient avoir développé une forme de communication et des pratiques propres à leur genre. Je ne fus pas déçue en arrivant à Lajamanu : les femmes étaient en pleine activité rituelle pour un cycle initiatique sacré Kajirri décrit vingt ans plus tôt comme réservé aux hommes (Meggitt, 1966) : en fait les deux sexes travaillaient rituellement de manière séparée mais explicitement complémentaire en se concertant régulièrement sur les rituels à faire. Dans un autre rituel intertribal et secret, que j'ai décrit comme " manifestation symbolique d'une transition économique » ou encore « culte du cargo » ou " culte historique » (Glowczewski, 2002, 2004), les femmes avaient les mêmes rôles que les hommes : et les deux sexes étaient initiés ensemble. En 1984, je retrouvais la même effervescence rituelle des femmes et des hommes. L'apprentissage de la langue warlpiri me permit d'enregistrer près de 90 heures de récits mythiques et de chants rituels correspondants et de révélations oniriques, tout en photographiant les peintures et danses associées à ces mêmes parcours mythiques de site en site totémique. L’analyse des chants et des peintures me révéla la spécificité de chaque univers totémique et leur logique de liens dans une cosmologie extrêmement complexe et dynamique faite de singularités entrecroisées.

Pour comprendre ce que j'avais partagé avec les Warlpiri sur le terrain, j'ai d'abord cherché à modéliser avec une figure topologique - l'hypercube - leur manière de se situer dans un réseau classificatoire à huit pôles. Les 8 sous-sections des noms dits de " peau » (skin names) qui sont énoncés comme un modèle de l'organisation sociale et de la vie rituelle sont démultipliées en une multitude d'agencements, que les Warlpiri (et leurs voisins du désert) cartographient eux-mêmes dans la géographie physique du désert et de ses sites sacrés. A cette géographie correspond une géographie spirituelle des récits mythiques des êtres totémiques et des chants rituels qui relient ces sites comme des balises. Ces balises à toponymes fonctionnent comme des empreintes, engrammes d’une mémoire vivante, à la fois passé idéalisé et virtualité en potentiel de nouveaux événements (naissances, morts, alliances, conflits, phénomènes climatiques, etc.), notamment par l'interprétation de certains rêves, révélés tant aux femmes qu'aux hommes, pour continuer à relier les sites par des récits, des chants et des peintures rituelles correspondantes et localiser les référents totémiques de chaque nouveau-né. 
Dans cette approche cartographique d'expériences matérielles et immatérielles, actuelles et virtuelles, les explications aborigènes des rites, des mythes et des rites étaient indispensables : il s'agissait pour moi de montrer comment les Aborigènes du désert fabriquent du lien social et spirituel entre les hommes, avec les sites et les héros totémiques de ce réseau. Leurs explications et la traque systématique dans la littérature australienne, alors très abondante, des divers tabous rencontrés me permirent de dresser une sorte de matrice croisée sur les contextes et les domaines de leur applications : d'une part les tabous étaient langagiers, spatiaux, sexuels ou relatifs aux biens, notamment alimentaires : d'autre part ils s'appliquaient presque toujours à l'occasion de quatre types de rituels différents : totémiques, de deuil, d'initiation ou de règlement de conflit entre alliés.

Autrement dit, si quelque chose de normatif - tabous et prescriptions totémiques des noms de peau - permettait la reproduction du " système ", ce que les Aborigènes du désert appellent la Loi, ce n'était qu'à la condition que tous les hommes et femmes du groupe linguistique continuent de faire des rituels en les « réinterprétant », c’est-à-dire en rêvant des révélations qui nourrissent cette reproduction. Ces révélations - perçues comme des virtualités de l'espace-temps du rêve remémorisées par le rêveur, se présentaient sous forme d'innovations individuelles transposées dans des formes pour nous artistiques (peintures, chants, danses) dans lesquelles le groupe se « reconnaît ». Durkheim aurait peut-être trouvé là une confirmation de sa thèse sur l'idéal collectif et le statut du groupe mais Tarde aussi sur l'imitation et l'innovation comme ondes de propagation qui traversent les individus, créant des territoires existentiels dont les agencements se recomposent sans cesse (Guattari, 1992 ; Guattari ; Glowczewski, 1987). Deleuze (1993, p. 83 citant en note Glowczewski, 1991) a reconnu ce processus de gestion collective des rêves ancrés dans les parcours géographiques qui fabriquent des cartes à la fois imaginaires et réelles :

C'est pourquoi l'imaginaire et le réel doivent être plutôt comme deux parties juxtaposables ou superposables d'une même trajectoire, deux faces qui ne cessent de s'échanger, miroir mobile. Ainsi les aborigènes d'Australie joignent des itinéraires nomades et des voyages en rêve qui composent ensemble un « entremaillage de parcours » « dans une immense découpe de l'espace et du temps qu'il faut lire comme une carte ».

Horizontes Antropológicos, Porto Alegre, ano 20, n. 41, p. 381-403, jan./jun. 2014 


\section{Histoire et anthropologie : patrimonialisation vs réappropriations autochtones}

En 1991, un nouveau terrain sur la côte nord-ouest de l'Australie, me fit découvrir des groupes en recomposition constante entre descendants d'Aborigènes reconnus gardiens des lieux et d'autres exilés de leurs terres d'origine. Il devaient sans cesse prouver leur légitimité face aux injonctions gouvernementales qui, après des décennies de déplacements forcés de leurs parents ou grands-parents dans les missions et réserves, exigeaient pour la reconnaissance de leurs droits qu'ils démontrent une continuité culturelle comme si ces aléas de l'histoire n’avaient pas existé. Les Aborigènes semblaient eux ne tenir que par l'alternance d'alliances et de conflits qui les opposaient dans le cadre même de l'Etat australien. Il n'y avait plus de société aborigène palpable et pourtant, si ceux qui se disaient aborigènes, métis compris, vivaient en apparence comme les Blancs, ils valorisaient aussi leurs différences comme un « système » qui aurait sa place souveraine au sein de la nation australienne alors même qu'ils étaient rejetés pour d'autres différences interprétées comme un « système » inconciliable avec les impératifs universalistes énoncés par l’Etat.

Depuis la fin des colonisations officielles, la planète ne semble plus autoriser l'existence de sociétés coupées du reste du monde, mais les cultures issues de ces sociétés cherchent à se mémorialiser dans des formes créatives de patrimonialisation. Les Etats occidentaux encouragent ce processus à condition que la culture s'en tienne à la "représentation » de son passé et d'un espace clos restreint à la famille, au voisinage (si pas trop communautariste) ou au spectacle. Or pour les acteurs concernés, la culture n’a de sens que si elle devient le socle même des échanges sociaux et politiques. Les Aborigènes du désert ont pensé réussir cet " échange » avec leur mouvement de peintures à l'acrylique sur toile (transposant leurs réseaux territorialisés de mythes et itinéraires totémiques) qui sont entrés en force sur le marché de l'art contemporain. Trente ans plus tard, les collections de leurs oeuvres ont acquis une grande valeur en bourse, mais la souveraineté aborigène sur leurs terres est écrasée au jour le jour et les résistants aux injustices sociales souvent muselés (Glowczewski, 2012). Partout en Australie des courants de peinture ont émergé avec quelques artistes qui ont atteint une renommée mondiale : la plupart de ceux qui proviennent des communautés appuient leur art sur des références totémiques et territoriales comme une affirmation existentielle, une ontologie qui relie les humains à tout ce qu'ils ont nommés. 
Il reste donc qu'en l'absence de " sociétés » aborigènes bien des individus ou collectifs aborigènes s'attachent à certaines formes autrefois décrites comme du "totémisme" (esprit-enfant, lien spirituel à une terre, etc.) : s’agit-il de formes élémentaires de la vie religieuse? Pour beaucoup cette spiritualité se marie avec l'une ou l'autre des églises chrétiennes, et pour d'autres, elle se conjugue avec leurs convictions laïques ou musulmanes. En ce sens, ces singularités existentielles aborigènes plutôt que de fonder des prémisses religieux apparaissent comme des formes élémentaires de ce que Guattari (1989, 1992) a appelé l'écosophie (nouage de trois écologies : mentale, sociale et environnementale), un paradigme esthétique qui est à la fois éthique et politique, un ancrage nomade de survie.

Un récent colloque consacré à « 1913. La recomposition de la science de l'homme » rendait compte d'un projet ANR de patrimonialisation des « savoirs ethnographiques ». Lors d'une discussion, alors que j'avais questionné la pertinence d'interpréter les débats anciens sur le totémisme sans prendre en compte la parole des Aborigènes qui se sont exprimés depuis, l'un des intervenants me répondit que les Aborigènes d'aujourd'hui n’ont (je cite) « rien à dire sur ce qui se disait d'eux à l'époque car ce ne sont plus les 'mêmes' ". Vieille rengaine de l'authenticité et de la légitimité du lieu d'énonciation d'un savoir. Je racontais alors qu'en 2001, le musée du Victoria à Melbourne a intégré aux côtés d'objets d'Australie centrale collectés par Gillen et présentés dans l'exposition permanente, une installation sur deux écrans intitulée The dialogue : d'un côté un acteur joue le rôle de l'anthropologue Baldwin Spencer (Spencer; Gillen, 1899) qui, avec les arguments et préjugés de son époque, dialogue avec un acteur aborigène qui, sur l'autre écran, interprète Irrapmwe, son principal informateur arrernte. Mais ce dernier remet en question ou précise un certain nombre d'affirmations de Spencer en utilisant des arguments éthiques de ces dernières décennies, fondés sur la prise de parole et les droits autochtones de propriété collective et inaliénable de leurs terres et de la propriété intellectuelle de leur savoirs traditionnels (Morton, 2004). Frédérico Rosa (2012) me répondit alors « vraiment ces Australiens sont trop politiquement corrects!».

Ce court-circuitage d'un siècle utilisé à des fins pédagogiques dans un musée peut apparaître comme un effet de style facile mais il pointe une question essentielle : le rapport politique à l'histoire des idées. En effet à l'époque de Durkheim qui utilisa les écrits quasi contemporains de Spencer et Gillen

Horizontes Antropológicos, Porto Alegre, ano 20, n. 41, p. 381-403, jan./jun. 2014 
pour rédiger Les formes élémentaires de la vie religieuse certains faits ethnographiques qu'ils ont relevés sont restés sans commentaire car le paradigme occidental d'alors ne permettait pas de les comprendre. Il a fallu que nous changions de perception et de paradigme pour voir et comprendre autrement les données : particulièrement, en ce qui concerne le rapport spirituel à la terre pensé comme un réseau de lieux inter-reliés, en devenirs mouvants dans un espace-temps qui se rapproche plus des théories de la physique quantique et du cyberespace que des spéculations religieuses à l'origine de nos disciplines écartelées dans les oppositions entre individu et société ou nature et culture (Descola, 2005).

Certes du point de vue de l'épistémologie des sciences, les savoirs ethnographiques sont à préserver mais il serait étrange de patrimonialiser l'anthropologie - même française - sans prendre en compte les savoirs tels qu'exprimés par les populations concernées par ces études depuis la fondation de la discipline et particulièrement avec l'indépendance et les revendications territoriales et de souveraineté de ces peuples qui furent colonisés et abondamment ethnologisés (Langton, 2011; Toussaint, 2006). En l'occurrence le fait que Durkheim a projeté la notion de moitiés totémiques dans une fausse division de l'espace territorial en deux est-il encore un « savoir »? Pris dans le dualisme de son époque, il ne pouvait sans doute visualiser la territorialité aborigène dans un espace réticulaire. ${ }^{4}$ Or, les Aborigènes ont élaboré leur totémisme rhizomique à l'image des rhizomes d'ignames qui parcourent le désert; soit un de leur modèle à penser qui - comme je l'ai souligné ailleurs - n'est devenu un savoir visible pour l'Occident qu'à partir du moment où nous nous sommes familiarisés avec des théories de flux et de rhizomes telles celles développées par Tarde, puis Simondon, Deleuze et Guattari, et qui ont trouvé écho dans le développement réticulaire de l’internet des années 1990 (Glowczewski, 2007).

Penser le totémisme australien comme une cartographie rhizomique ou réticulaire d'intersubjectivation des humains et de tout ce qui est nommé

4 Dans une note des Formes élémentaires, Durkheim signale que les totems peuvent parfois être des lieux, sans mesurer le sens des descriptions totémiques de Spencer et Gillen (1899) qui eux-mêmes n'ont pas mesuré l'importance du fait que chez les peuples du désert et du nord tous les totems - animaux, plantes, vent, pluie, mais aussi lance, objets, etc. - sont dans un certain rapport d'identification avec plusieurs lieux reliés par leurs mythes respectifs.

Horizontes Antropológicos, Porto Alegre, ano 20, n. 41, p. 381-403, jan./jun. 2014 
déplace la discussion telle que posée dans le texte co-écrit par Durkheim et Mauss (1903) « De quelques formes primitives de classification ». Ce texte, qui préfigurait Les formes élémentaires de la vie religieuse, interrogeait l'essence et la hiérarchie entre les différents totems auxquels une personne dit s'identifier. Or plutôt que de classer et sous-classer, il s'agit de comprendre les formes de devenir (en warlpiri exprimé par le postfixe jarri) qui lient les humains (hommes et femmes) aux différents totems dont ils portent le nom, en interaction avec les responsabilités rituelles et leurs rôles de gardiens fonciers qui varient selon les contextes. Les formes de devenir totémiques sont ainsi fluctuantes, à la fois spirituelles dans les rites et tangibles dans les corps, les animaux, les plantes, la terre, l'eau, le vent, etc. sous forme de traces, d'empreintes, de forces vitales. Empreintes comme des « gènes » disent certains Aborigènes aujourd'hui (en Warlpiri « kuruwarri ») sans entendre la transmission des " gènes » au sens d'une filiation exclusive, endogame ou raciale. Au contraire, quand un Aborigène dit « je suis » Opossum, Prune et Graine mais aussi tel ou tel site, la qualité commune n'est pas tant une essence qu'une empreinte, combinable avec d'autres empreintes, et qui porte une virtualité de devenir commun nouant le possible des humains avec tout le reste. Par définition, on reproduit un totem en se mariant avec quelqu'un qui incarne une constellation de totems autres que les siens. L'exogamie totémique pose ainsi l'altérité comme condition de la filiation qui reproduit un système dynamique d'alliances.

J'avais commencé ma communication orale au colloque du centenaire Durkheim en retraçant au feutre le dessin réalisé dans le sable par Wanta Steven Patrick Jampijinpa, ancien instituteur warlpiri à Lajamanu, concepteur du festival Milpirri, qui est depuis 2012 chercheur honoraire dans un programme de recherche sur les chants warlpiri à Canberra. J'invite les lecteurs à voir par eux-mêmes sur YouTube (Jampijinpa, 2006), le clip de Wanta qui commente son dessin tout en le traçant avec un bâton sur le sable, puis reprenant dans une classe d'école, en montrant sur une peinture colorée un autre réseau correspondant au découpage en quatre ensembles totémiques.

Le dessin de Wanta est une manière warlpiri de répondre à la divergence entre Durkheim et Spencer et Gillen tel qu'analysé par William Watts Miller (2012) au colloque parisien du centenaire des Formes : ces derniers séparaient totémisme et organisation sociale alors que Durkheim les réassociait mais en séparant sacré et profane. Wanta, le Warlpiri, ne se situe pas dans le dualisme

Horizontes Antropológicos, Porto Alegre, ano 20, n. 41, p. 381-403, jan./jun. 2014 
ou la dualité de telles catégories. Son cadre de référence est un réseau qui relie entre eux ce qu’il désigne en anglais comme cinq pillars, « piliers » : 1) ngurra la terre comme camp, site, " chez soi », 2) kurruwari, le dreaming qui est et fait la Loi de tous les devenirs, 3) jardiwanpa, etc.... toutes les différentes cérémonies dont les rituels, chants, danses, peintures réactualisent les liens,; 4) la langue warlpiri; 5) walja, les relations de parenté, qui associent les gens deux à deux dans un jeu de relations correspondant à un groupe diédrique (le cube : modèle logique du fameux système dit à « classes » qui a fait couler tant d'encre chez les anthropologues comme chez les mathématiciens.

Les relations de ce cube ont érigé les Aborigènes comme le modèle exemplaire du structures élémentaires de la parenté, mais les recherches de Fred Myers auprès des "Pintupi » qui ne pratiquaient pas ce système classificatoire jusqu'aux années 1930, lui a fait dire que les Pintupi seraient phénoménologiques, là où les Warlpiri seraient structuralistes. Certes, il y a des aspects fort différents dans l'organisation des modes d'existence des Warlpiri et des Pintupi, mais aussi diverses formes d'interactions (l'adoption du système des sous-sections par le Pintupi dans les années 30 et divers échanges rituels, intermariage, etc.) qui réactualisent leurs singularités respectives au-delà de l'opposition phénoménologique/structurale. L'avènement des peintures sur toile sur le marché de l'art contemporain initié par les Pintupi, les opposa dans un débat d'experts rituels avec les Warlpiri. Le conflit s'est d'une certaine manière résolu en faisant changer de pratique les uns et les autres : Les Warlpiri s'opposaient à ce que soient peints pour le publics le motifs totémiques sacrés - dont les Pintupi, Warlpiri et autres groupes du désert partagent les itinéraires des Dreamings. Pour tous, ces motifs relèvent d'un processus d'initiation interne (bien que commun et objet d'échange entre la plupart des groupes du désert et au-delà entre initiés, hommes ou femmes). Les Pintupi ont accepté de changer la manière dont ils avaient commencé à peindre afin de rendre les motifs moins explicitement rituels, en systématisant sur les fonds des toiles des textures de points (qui renvoient par ailleurs aux kuruwarri, et duvet ou coton sauvage des peintures corporelles masculines) et en supprimant les références figuratives aux objets sacrés et aux personnes (Myers, 2002). Les Warlpiri, qui avaient été choqués par l'exposition des premières peinture sur toile pintupi qu'ils avaient vu à Paris en 1983, se sentirent ainsi encouragés à peindre à leur tour - sans risquer un sacrilège - (Glowczewski, 2004). 
Lorsqu'en 2005 YouTube a permis de poster des images sur le WEB gratuitement, les Aborigènes se sont lancés avec enthousiasme; Wanta a alors aussi conçu un festival, Milpirri, où les enfants dansent du hip hop à côté des anciens qui chantent et dansent les voyages mythiques des héros totémiques, les images-forces kuruwarri : c'était sa réponse au suicide des jeunes. ${ }^{5}$ Les adultes et les enfants portent des vêtements soient jaune, soit bleu, soit rouge soit vert, codes de couleur servant à répartir les différents kuruwarri, les totems avec leurs lieux et rituels associés, selon les quatre paires de "classes » (sous-sections) que tous les Aborigènes du désert et du nord appellent en anglais des skin names, «noms de peau ». Le réseau des quatre couleurs et cinq piliers de Wanta n'est ni structuraliste, ni phénoménologique, il est plutôt une « invention » au sens tardien, traduisant une micropolitique devenue écosophique (Guattari 1989, 1992) : celle d'un Aborigène qui, de colonisé par naissance, s'est singularisé dans une ligne de fuite, en fabriquant une innovation collective, en cristallisant les diverses transformations et difficultés que son peuple a subies depuis la sédentarisation forcée en réserve dans les années 1950 jusqu'à l'intervention en 2007 de l'Etat qui a interrompu le principe d'autogestion instauré par les Warlpiri qui avaient gagné en 1978 leur revendication territoriale sur leurs terres spoliées.

Les savoirs des sociétés d'Orient à « érudits » dont les paroles ont été fixées par écrit se sont fait une place dans le champ universel des sciences, mais il encore de bon ton dans certains milieux de rejeter les savoirs d'autres peuples sous prétexte qu'ayant été « sans écriture » avant leur colonisation, ils auraient été jusque là « sans histoire » et seraient condamnés à ne pas changer - ni s’autoriser à interpréter leur histoire et la cosmologie de leurs sociétés sauf à perdre non seulement leur authenticité, mais leur simple droit d'exister comme différent et singulier. Or, les manières actuelles dont des membres de ces peuples s'expriment et interprètent leurs savoirs sont en train de défier à la fois le sens de l'anthropologie et la légitimité supposée des savoirs occidentaux.

5 http://www.documentaryaustralia.com.au/films/details/1551/milpirri-winds-of-change.

Horizontes Antropológicos, Porto Alegre, ano 20, n. 41, p. 381-403, jan./jun. 2014 


\section{Devenirs}

Durkheim (2013, p. 3) écrivait dans Les formes élémentaires de la vie religieuse :

Les rites les plus barbares ou les plus bizarres, les mythes les plus étranges traduisent quelque besoin humain, quelque aspect de la vie soit individuelle soit sociale. Les raisons que le fidèle se donne à lui-même pour les justifier peuvent être, et sont même le plus souvent, erronées; les raisons vraies ne laissent pas d'exister; c'est affaire à la science de les découvrir.

L'enjeu pour Durkheim étant de trouver des lois qui puissent s'appliquer à toutes les sociétés au-delà des spécificités locales, mais est-ce à dire que les producteurs de ces rites et mythes ne peuvent pas aussi contribuer à cette quête supposée réservée à la science?

Depuis une trentaine d'années, la prise de parole des peuples autochtones et la valorisation plus récente des systèmes de savoir indigènes (notamment avec la promotion du patrimoine immatériel à l'ONU) semblent rendre inactuelle la question de l'erroné et du vrai telle que posée par Durkheim à son époque. En effet, l'enjeu contemporain des sciences sociales - je dirais même leur priorité - plutôt que de découvrir " les raisons vraies » des rites et des mythes est plutôt de comprendre la cohérence de ce qui, selon les autochtones fait « système de savoir ». Système n'est pas entendu ici au sens d'une reproductibilité atemporelle, mais, de ce qui, tout en se référant à des savoirs hérités d'une tradition (y compris la scientifique), résiste comme agencement dynamique permettant aux acteurs collectifs de « tenir » une singularité.

Celle-ci peut souvent ne tenir qu'à se reconstruire régulièrement, ou selon les contextes, par-delà les contingences historiques et la violence des transformations sociales (économiques, politiques, environnementales, et aussi psychiques, religieuses) que les groupes concernés (ethniques ou chercheurs de telle ou telle école) ont subi avec la colonisation jusqu'aux flux constants de la globalisation actuelle. Face à cet impératif, ethnographier les réponses les plus singulières ne nous voue pas au relativisme culturel mais au contraire à tenter de comprendre des formes transversales à l'espace et au temps à l'œuvre dans ce qui non seulement maintient ces singularités culturelles mais encore en fait émerger de nouvelles. 
Les sciences sociales - et particulièrement l'anthropologie - sont face à une impasse si elles continuent à se patrimonialiser par la seule voix de ceux qui se légitiment de la "filiation" de l'histoire de nos disciplines en intégrant les transformations de la pensée occidentale dans une continuité fictive qui ne reconnaît pas l'apport de la performativité des “étudiés” et les changements de paradigme qu'ils ont induits (Glowczewski, 2012). Je laisse le dernier mot à un jeune artiste aborigène du sud de l'Australie mondialement acclamé, Brook Andrew (2013, p. 246) :

Arguably, evolutionary theory is a backward invention fraught with confusion made into an absolute rule on how the 'other' is seen. Therefore this means nothing to me or my family apart from the fact that we still cannot own our grandmothers traditional lands due to invasion/colonization. The idea of the primitive is no more than a European fantasy - my ancestors were 'mere uncivilized creatures who had no real human meaning in life'. This objectification is an insult to all humanity. This type of theory is redundant and never had any real truth other than a Western European ego and power over those who were not a mirror image of Emile Durkheim.

\section{Références}

ANDREW, B. Entretien réalisé par Arnaud Morvan. In: MORVAN, A. (Ed.). Mémoires vives: une histoire de l'art aborigène. Bordeaux: Musée d'Aquitaine et Paris; Editions de la Martinière, 2013. p. 104-105. Catalogue bilingue d'exposition.

DELEUZE, G. Foucault - Le Pouvoir cours 9. La voix de Gilles Deleuze en ligne, 7 janv. 1986. Université Paris 8, audio et transcription en ligne. Disponible sur: <http://www2.univ-paris8.fr/deleuze/article.php3?id_ article=439>. Accédé: 30 août 2013.

DELEUZE, G. Critique et clinique. Paris: Minuit, 1993.

DELEUZE, G.; GUATTARI, F. Mille plateaux. Paris: Minuit, 1980.

DESCOLA, P. Par delà nature et culture. Paris: Gallimard, 2005. 
DURKHEIM, E. Les formes élémentaires de la vie religieuse. 7e éd. Paris: PUF, 2013. (Collection Quadrige).

DURKHEIM, E.; MAUSS, M. De quelques formes primitives de classification, Contribution à l'étude des représentations collectives (1901-1902). Année sociologique, Paris, v. 6, p. 1-72, 1903.

FREUD, S. Totem et tabou. Paris: Petite Bibliothèque Payot, 1975.

GLOWCZEWSKI, B. Du rêve à la loi chez les Aborigènes: mythes, rites et organisation sociale. Paris: PUF, 1991. Disponible sur: <https://repositorio. ufsc.br/xmlui/handle/123456789/99708>. Accédé: 30 août 2013.

GLOWCZEWSKI, B. Culture Cult. The ritual circulation of inalienable objects and appropriation of cultural knowledge (North-West Australia). In: BALLINI, M. J.; JUILLERAT, B. (Ed.). People and things: social mediation in Oceania. Durham: Carolina Academic Press, 2002. p. 265-288.

GLOWCZEWSKI, B. 2004 Rêves en colère. Avec les Aborigènes australiens. Paris: Plon, 2004. (Terre Humaine).

GLOWCZEWSKI, B. La pensée en réseau des Aborigènes. In: DANIEL, J. Le siècle de Lévi-Strauss. Paris: CNRS Editions, 2005. p. 149-155.

GLOWCZEWSKI, B. Linhas e entrecruzamentos: hiperlinks nas narrativas indigenas australianas. In: GROSSI, M.; ECKERT, C.;FRY, P. (Org.). Conferências e diálogos: saberes e práticas antropológicas. Blumenau: Nova Letra, 2007. p. 181-201.

GLOWCZEWSKI, B. Entre o espectáculo e a política: singularidades indígenas. Cadernos de Subjetividade, n. 13, p. 120-142, 2011 a.

GLOWCZEWSKI, B. Guattari and Anthropology. In: ALLIEZ, E.; GOFFEY, A. (Ed.). The Guattari Effect. London: Continuum Books, 2011b. p. 99-114.

GLOWCZEWSKI, B. From academic heritage to Aboriginal priorities: anthropological responsibilities. R@U: Revista de Antropologia da UFSCar, v. 4, n. 2, p. 6-19, jul./dez. 2012. Disponible sur: <http://issuu.com/raufscar/ docs/r_uv4n2?e=2030228\%2F3965435>. Accédé: 30 août 2013. 
GUATTARI, F. Trois écologies. Paris: Galilée, 1989.

GUATTARI, F. Chaosmose. Paris: Galilée, 1992.

GUATTARI, F.;GLOWCZEWSKI, B. « Les Warlpiri » discussion avec Felix Guattari (1983 et 1985). Chimères, n. 1, p. 4-37, p. 1987.

GUATTARI, F.; ROLNIK, S. Micropolítica: cartografias do desejo. Petrópolis: Vozes, 1986.

JAMPIJINPA, W. S. P. Ngurra-kurlu. [2006]. Disponible sur: <http://www. youtube.com/watch?v=iFZq7AduGrc\&feature=related $>$. Accédé: 30 août 2013.

LANGTON, M. Anthropology, politics and the changing world of Aboriginal Australians. Anthropological Forum, v. 21, n. 1, p. 1-22, 2011.

LATOUR, B. Gabriel Tarde and the End of the Social. In: JOYCE, P. (Ed.). The social in question: new bearings in history and the social sciences. London: Routledge, 2002. p. 117-132.

LATOUR, B. Formes élémentaires de la sociologie; formes avancées de la théologie. Paris, 2012. Contribution pour le colloque du centenaire Collège de France, sous la direction de Frédéric Keck et Perig.

LAZARATTO, M. Postface. Gabriel Tarde: un vitalisme politique In: TARDE, G. Monadologie et sociologie. Paris: Les empêcheurs de penser en rond, 1999. p. 103-150.

LAZARATTO, M Puissances de l'invention: la psychologie économique de Gabriel Tarde contre l'économie politique. Paris: Les Empêcheurs de penser en rond, 2002.

MYERS, F. R. Painting culture: the making of an Aboriginal Hight Art. Durham: Duke University Press, 2002.

MEGGITT, M. Gadgeri among the Warlpiri Aborigines of Central Australia. Sydney: The University of Sydney, 1966. (Oceania Monograph, 14). 
MORTON, J. 'Such a man would find few races hostile': History, fiction and anthropological dialogue in the Melbourne Museum. Arena Journal, n. 22, p. 53-71, 2004.

ROSA, F. Le totémisme hier: obsessions marginales d'un débat anthropologique. 2012. Communication au colloque 1913. La recomposition de la science de l’homme, Paris MNHN, 15-16 mars.

SIMONDON, G. L'individuation à la lumière des notions de forme et d'information. Paris: PUF, 1964.

SPENCER, W. B.; GILLEN, F. J. Native tribes of Central Australia, 1899.

TOUSSAINT, S. A time and place beyond and of the center: Australian anthropologies in the process of becoming. In: RIBEIRO, G. L.; ESCOBAR, A. (Ed.). World anthropologies: disciplinary transformations within systems of power. Oxford: Berg, 2006. p. 225-238.

WATTS MILLER, W. Durkheim's re-imagination of Australia: a case study of the relation between theory and "facts". L'Année sociologique, v. 62, n. 2, p. 329-349, 2012. 